\title{
Recent Studies of Rubberlike Elasticity
}

\author{
James E. MARK \\ Department of Chemistry and the Polymer Research Center, \\ The University of Cincinnati, Cincinnati, Ohio 45221, U.S.A.
}

(Received August 20, 1984)

\begin{abstract}
Forming elastomeric networks by end linking functionally-terminated polymer chains has the advantage of minimizing imperfections in the network structure, with associated improvements in ultimate properties. These improvements are found to be particularly marked when mixtures of very short and relatively long chains are end linked, thus giving bimodal networks. Such (unfilled) networks show very large increases in reduced stress or modulus at high elongations and, since such a toughening effect is of interest with regard to both fundamental questions and potential applications, a variety of experiments were carried out to elucidate its origin. These experiments included stress-strain isotherms over a wide range in temperature and degree of swelling, stress-temperature coefficients, and birefringence-temperature coefficients. All of the experimental results indicate the origin of the upturns in modulus to be intramolecular, specifically non-Gaussian effects arising from the very limited extensibility of the short chains present in the networks. This limited extensibility and its effects on elastomeric properties were investigated using a theory of rubberlike elasticity based on network distribution functions generated from rotational isomeric state models for the specific chains of interest. Polymers studied to date by this theoretical approach are poly(dimethylsiloxane), (amorphous) polyethylene, polymeric sulfur, and polymeric selenium.
\end{abstract}

KEY WORDS Rubberlike Elasticity / Model Networks / Bimodal Networks / Non-Gaussian Theory / Ultimate Properties / Rotational Isomeric State Theory / Poly(dimethylsiloxane) / Polyethylene / Polymeric Sulfur / Polymeric Selenium /

The elastomeric properties of a network of flexible polymer chains obviously depend on the structure of the network, in particular its number density of cross links. ${ }^{1,2}$ A more subtle, but nonetheless very important dependence is the relationship between such properties and the spacing of the cross-links along the chains, i.e., the network chain length distribution. ${ }^{3}$ There has been very little experimental information relevant to this issue, simply because the usual methods ${ }^{1,4}$ for preparing elastomeric networks do not permit control of the chain length distribution, and there are no experimental techniques for determining it once the network is formed.

Newly developed techniques, however, now do permit this control by restricting the cross-linking reaction to specific groups located at the chain ends or at a known average spacing along the chains. ${ }^{5-8}$ Thus, if functionally terminated chains are end linked into a network structure, then the distribution of network chain lengths is essentially identical to that of the uncross-linked chains from which it was prepared. An additional advantage is the minimization of imperfections in the network structure, with corresponding improvements in ultimate properties. $^{9}$

An important example of this new approach is the end linking of a mixture of very short and relatively long chains to give a bimodal network..$^{9,10}$ Such (unfilled) networks have unusual elastomeric properties, in particular high extensibilities and large increases in modulus at high elongations. These increases are of fundamental interest because they represent marked deviations from the usual Gaussian theories ${ }^{1,2}$ of rubberlike elasticity. They are also of practical importance since they constitute significant improvements in the ultimate properties of an elastomer. 
The present review provides information on (i) the magnitudes of such strengthening. effects, (ii) their dependence on composition, (iii) their molecular origin, and (iv) their interpretation in terms of a new non-Gaussian theory of rubberlike elasticity.

\section{EXPERIMENTAL RESULTS}

The elastomeric quantity of primary interest is the reduced stress or modulus ${ }^{9,11}$

$$
[\mathrm{f} *] \equiv f^{*} /\left(\alpha-\alpha^{-2}\right)
$$

where $f^{*} \equiv f / A^{*}$ is the nominal stress, $f$ the equilibrium elastic force, $A^{*}$ the undeformed crosssectional area of the sample, and $\alpha=L / L_{i}$ the relative length or elongation. It is generally plotted as a function of reciprocal elongation as suggested by the semi-empirical equation of Mooney and Rivlin $^{2,12}$

$$
\left[\mathrm{f}^{*}\right]=2 C_{1}+2 C_{2} \alpha^{-1}
$$

where $2 C_{1}$ and $2 C_{2}$ are constants independent of elongation.

Some illustrative isotherms ${ }^{13}$ for bimodal networks of poly(dimethylsiloxane) (PDMS) $\left[-\mathrm{Si}\left(\mathrm{CH}_{3}\right)_{2} \mathrm{O}-\right]$ are shown in Figure 1. The very large increases in the modulus at high elongations are due to the limited extensibility of the very short network chains. ${ }^{9,14,15}$ As shown here ${ }^{13}$ and elsewhere, ${ }^{16,17}$ the increases are largest at approximately $95 \mathrm{~mol} \%$ short chains; above this amount the networks are too brittle, and below it there are too many long chains capable of deforming in preference to the short chains.

A variety of experiments ${ }^{10,18,19}$ were carried out to test the assumption that the increases in modulus were due to the limited extensibility of the short chains, an exclusively intramolecular effect. The effect of temperature on the stress-strain isotherms is of particular importance in regard to the alternative possibility of strain-induced crystallization in the network. Typical results of this type are shown in Figure $2 .{ }^{18}$ Temperature is seen to have little effect on the elongation at which the upturn in [ $\mathrm{f}$ ] becomes discernible, the elongation at rupture, and the magnitude of the increases in [ $\mathrm{f}^{*}$ ]. Similarly, swelling the networks does not diminish the upturns. ${ }^{19}$ These results thus support the conclusion that the anomalous behavior is not due to straininduced crystallization.

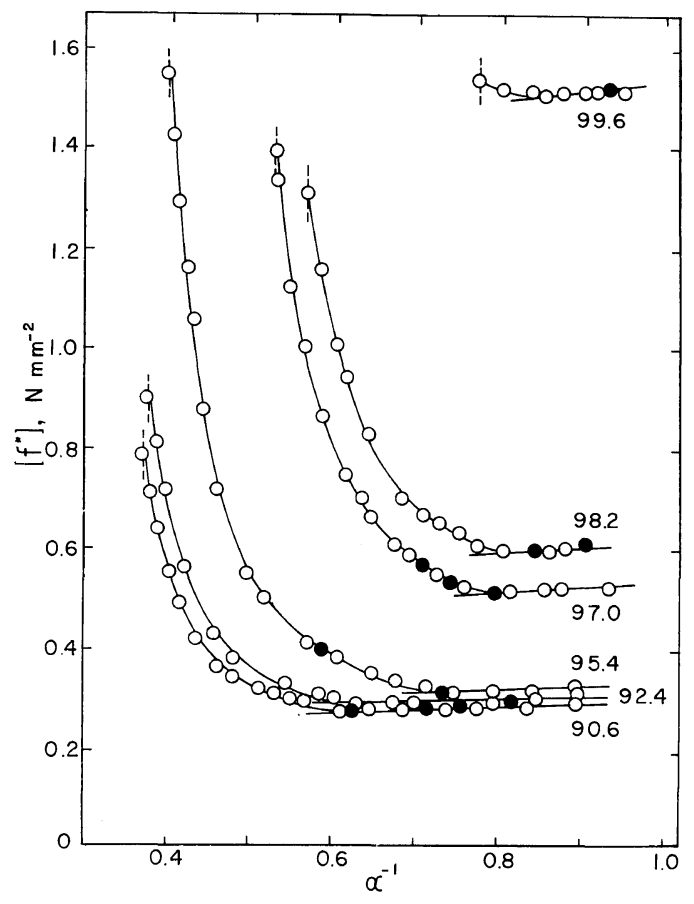

Figure 1. Typical stress-strain isotherms at $25^{\circ} \mathrm{C}$ for (unfilled) bimodal poly(dimethylsiloxane) networks in which the short and long chains have number-average molecular weights of 460 and $21: 3 \times 10^{3} \mathrm{~g} \mathrm{~mol}^{-1}$, respectively. ${ }^{13}$ Each curve is labelled with the $\mathrm{mol} \%$ of short chains present in the network. The filled circles correspond to results obtained out of sequence to test for reversibility, and the short extensions of the linear portions of the isotherms help locate the values of the elongation at which the upturn in modulus first becomes discernible. The vertical dashed lines locate the rupture points of the networks.

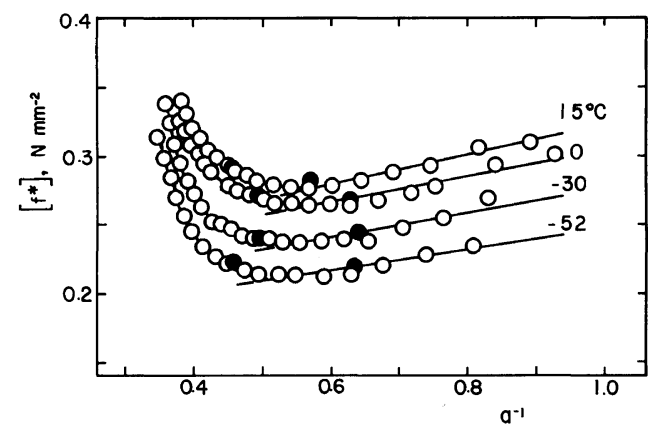

Figure 2. Typical results obtained to determine the effect of temperature on the stress-strain isotherms for a bimodal PDMS network (220 and $18.5 \times 10^{3} \mathrm{~g} \mathrm{~mol}^{-1}$ ) containing $75 \mathrm{~mol}^{\circ} \%$ of the very short chains. ${ }^{18}$ 


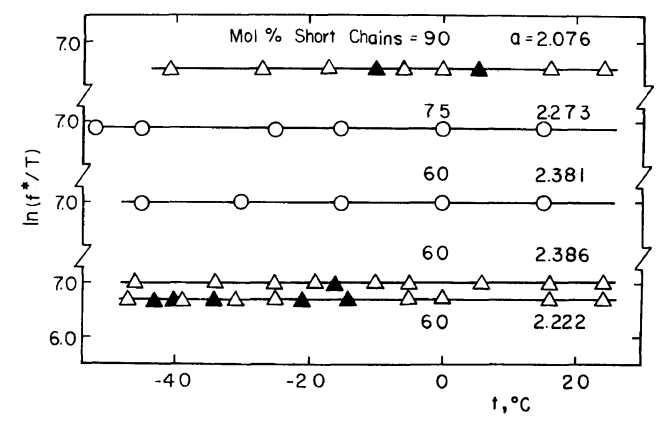

Figure 3. Representative thermoelastic data on a bimodal PDMS network (220 and $18.5 \times 10^{3} \mathrm{~g} \mathrm{~mol}^{-1}$ ), with circles referring to measurements at constant elongation and triangles to measurements of constant length. ${ }^{18}$ Filled symbols locate data obtained to check for reversibility.

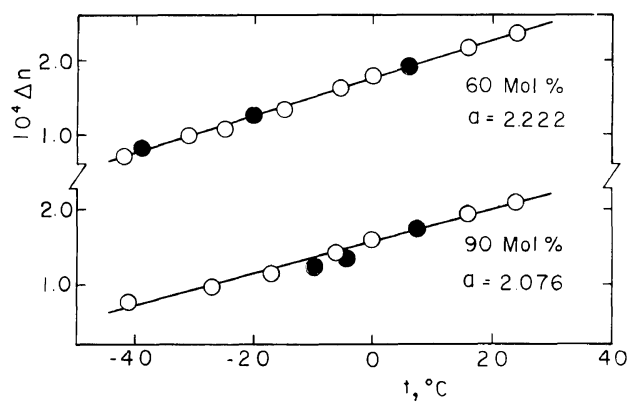

Figure 4. Typical birefringence-temperature relations on the $220,18.5 \times 10^{3} \mathrm{~g} \mathrm{~mol}^{-1}$ bimodal PDMS networks, with filled circles locating results obtained to check for reversibility. ${ }^{18}$

Also relevant here are some force-temperature ("thermoelastic") results ${ }^{18}$ obtained at elongations sufficiently large to give large increases in [ $\mathrm{f}^{*}$ ] in the stress-strain isotherm. Such curves, illustrated in Figure 3, show no deviations from linearity which could be attributed to strain-induced crystallization. Similarly, birefringence-temperature measurements show no non-linearity that could be attributed to crystallization, or to other intermolecular orderings of the network chains. Typical results of this type are shown in Figure $4 .{ }^{18}$

In practical terms, the cited results ${ }^{10,18,19} \mathrm{dem}$ onstrate that short chains of limited extensibility may be inserted in a long-chain network to improve its toughness. It is also possible to achieve the converse effect. Thus, bonding a small number of relatively long chains into a short-chain PDMS

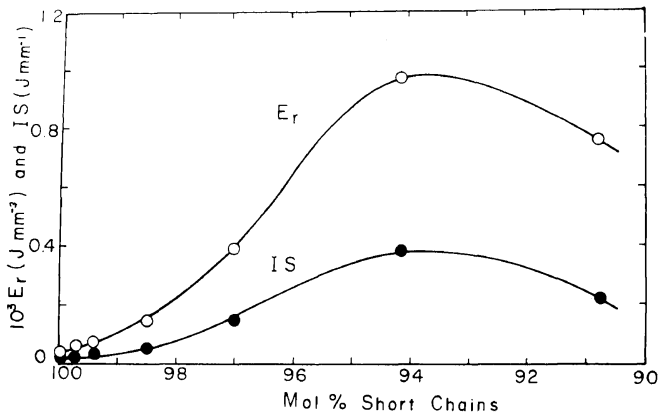

Figure 5. The energy required for rupture and impact strength (as measured in the falling-dart test) shown as a function of composition for $660,21.3 \times 10^{3} \mathrm{~g} \mathrm{~mol}^{-1}$ bimodal PDMS networks in the vicinity of room temperature. ${ }^{16}$

thermoset greatly improves its impact resistance, as is illustrated in Figure 5. ${ }^{16}$

\section{THEORY}

Since the above results demonstrate that the upturns in modulus are due to limited chain extensibility, it becomes important to try to interpret these results in terms of a non-Gaussian theory of rubberlike elasticity. The novel approach taken here utilizes the wealth of information which rotational isomeric state (RIS) theory ${ }^{20}$ provides on the spatial configurations of chain molecules. Specifically, Monte Carlo calculations based on the RIS approximation were used to simulate spatial configurations, and thus distribution functions for the endto-end separation $r$ of the chains. ${ }^{21-23}$ These distribution functions are used in place of the Gaussian function in the standard three-chain network mod$\mathrm{el}^{2}$ in the affine limit to give a molecular theory of rubberlike elasticity which is unique to the particular polymer of interest. ${ }^{14,15,24}$ Most important, it is applicable to the regions of very large deformation where limited chain extensibility gives rise to elastomeric properties significantly different from those in the Gaussian limit.

\section{CALCULATED RESULTS}

Some typical results calculated for polyethylene (PE) and PDMS chains having $n=40$ skeletal bonds are shown in Figure $6 .{ }^{14}$ The Gaussian distribution function is seen to be a relatively poor approxi- 


\section{J. E. MARK}

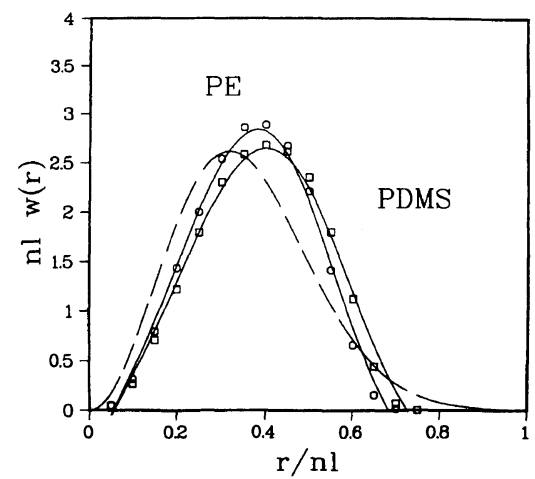

Figure 6. Comparisons among the rotational isomeric state (RIS) radial distribution functions at $413 \mathrm{~K}$ for PE ( $\bigcirc$ ) and PDMS $(\square)$ chains having $n=40$ skeletal bonds, and the Gaussian approximation ( - - ) to the PDMS distribution. ${ }^{14}$ The RIS curves represent cubic-spline fits to the discrete Monte Carlo data, for 80,000 chains, and each curve is normalized to an area of unity (with 1 being the skeletal bond length).

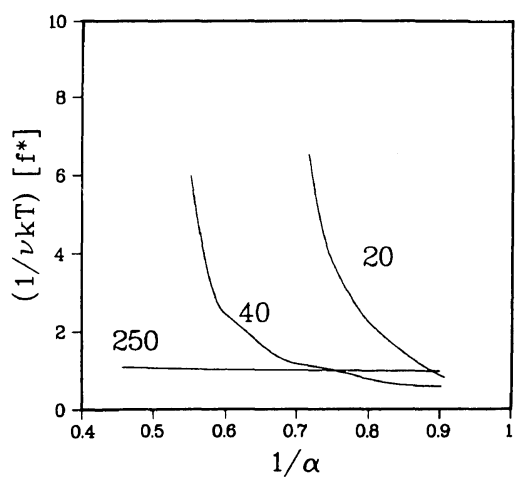

Figure 7. Moduli of PDMS networks having chains of lengths $n=20,40$, and 250 skeletal bonds. ${ }^{14}$ The values of $\left[\mathrm{f}^{*}\right]$ are normalized by the Gaussian prediction for the modulus, $v k T$, where $v$ is the number of network chains and $k T$ has its usual significance.

mation to the RIS distribution at this value of $n$ and becomes much worse as $n$ decreases. ${ }^{14}$ Calculated Mooney-Rivlin curves for networks made up of PDMS chains of various lengths are presented in Figure $7 .{ }^{14}$ As expected, the network of relatively long chains $(n=250)$ gives the Gaussian results $\left[\mathrm{f}^{*}\right] / v k T=1$. The upturns in [ $\left.\mathrm{f}^{*}\right]$ obtained at smaller $n$ are very similar to those found experimentally and illustrated in Figure 1. Also as expected, the results show that the shorter the network chains, the smaller the elongation at which the upturn occurs.

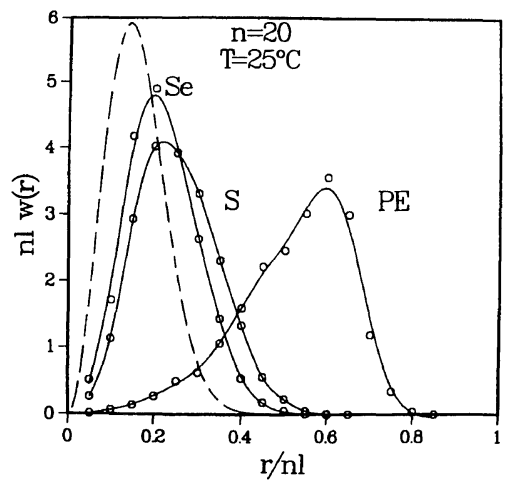

Figure 8. A comparison of the RIS distribution functions of $\mathrm{S}$ and $\mathrm{Se}$ with that for polyethylene (PE) for chains of 20 skeletal bonds at $25^{\circ} \mathrm{C}^{24}$ The dashed curve is the Gaussian approximation for the $\mathrm{S}$ chain.

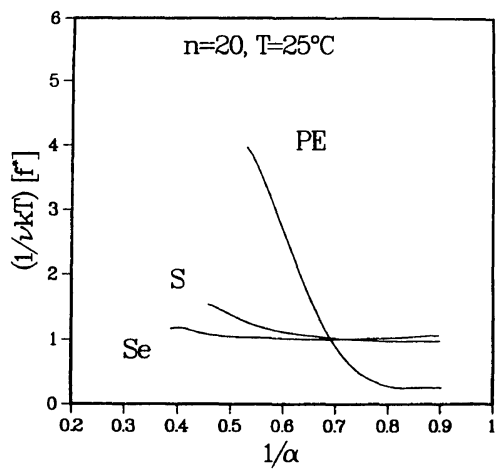

Figure 9. Calculated Mooney-Rivlin plots comparing networks of S and Se chains with that of PE chains for $n=20$, at $25^{\circ} \mathrm{C}^{24}$ Note that $\mathrm{S}$ and $\mathrm{Se}$ give almost Gaussian results in contrast to $\mathrm{PE}$, which shows a large upturn in [ $\left.\mathrm{f}^{*}\right]$.

The effects of chain flexibility on the distribution functions are illustrated in Figure 8. ${ }^{24}$ Sulfur and selenium chains are known to have extraordinary flexibility ${ }^{20}$ and this is reflected in the calculated distributions. Specifically the curves for $\mathrm{S}$ and Se are centered on values of the end-to-end separation which are much smaller than those for PE. The repercussions of this unusual flexibility on the stress-strain isotherms are shown in Figure $9 .{ }^{24}$ The curves for $\mathrm{S}$ and $\mathrm{Se}$ networks show only slight upturns in the modulus at high elongations. Large increases do occur in the results calculated for $\mathrm{PE}$ and PDMS,${ }^{14}$ as is illustrated by the curve for $\mathrm{PE}$ included in the figure. The results for $\mathrm{S}$ and $\mathrm{Se}$ 
networks are thus consistent with the unusually large flexibility of these chains. They thus clearly demonstrate how chain flexibility can have a profound effect on elastomeric properties, particularly in the region where limited chain extensibility can be very important.

Acknowledgements. It is a pleasure to acknowledge the financial support provided by the National Science Foundation through Grant DMR 79-18903-03 (Polymers Program, Division of Materials Research).

\section{REFERENCES}

1. P. J. Flory, "Principles of Polymer Chemistry," Cornell University Press, Ithaca, N. Y., 1953.

2. L. R. G. Treloar, "The Physics of Rubber Elasticity," Clarendon Press, Oxford, 1975.

3. F. Beuche, "Physical Properties of Polymers," Interscience, New York, 1962.

4. G. Odian, in "Elastomers and Rubber Elasticity," J. E. Mark and J. Lal, Ed., Am. Chem. Soc. Washington, D.C., 1982.

5. J. E. Mark, Makromol. Chem. Suppl., 2, 87 (1979).

6. J. E. Mark, Rubber Chem. Technol., 54, 809 (1981).

7. J. E. Mark, Pure Appl. Chem., 53, 1495 (1981).

8. P.-H. Sung, S.-J. Pan, J. E. Mark, V. S. C. Chang, J. E. Lackey, and J. P. Kennedy, Polym. Bull., 9, 375
(1983).

9. J. E. Mark, Adv. Polym. Sci., 44, 1 (1982).

10. J. E. Mark in "Elastomers and Rubber Elasticity," J. E. Mark and J. Lal, Ed., Am. Chem. Soc., Washington, D.C., 1982.

11. J. E. Mark and P. J. Flory, J. Appl. Phys., 37, 4635 (1966).

12. J. E. Mark, Rubber Chem. Technol., 48, 495 (1975).

13. J. E. Mark and M.-Y. Tang, J. Polym. Sci., Polym. Phys. Ed., 22, 1849 (1984).

14. J. E. Mark and J. G. Curro, J. Chem. Phys., 79, 5705 (1983).

15. J. G. Curro and J. E. Mark, J. Chem. Phys., 80, 4521 (1984).

16. M.-Y. Tang, A. Letton, and J. E. Mark, Colloid Polym. Sci., 262 (1984) in press.

17. M.-Y. Tang and J. E. Mark, Macromolecules $\mathbf{1 7}$ (1984) in press.

18. Z.-M. Zhang and J. E. Mark, J. Polym. Sci., Polym. Phys. Ed., 20, 473 (1982).

19. J. E. Mark, Macromolecules, 17 (1984) in press.

20. P. J. Flory, "Statistical Mechanics of Chain Molecules," Interscience, New York, 1969.

21. D. Y. Yoon and P. J. Flory, J. Chem. Phys., 61, 5366 (1974).

22. P. J. Flory and V. W. C. Chang, Macromolecules, 9, 33 (1976).

23. J. C. Conrad and P. J. Flory, Macromolecules, 9, 41 (1976).

24. J. E. Mark and J. G. Curro, J. Chem. Phys., 80, 5262 (1984). 